\title{
¿SUPONE EL CÁLCULO INFINITESIMAL UN ABANDONO DE LA METAFÍSICA?
}

\author{
Miguel Palomo \\ Universidad de Sevilla, España
}

\begin{abstract}
RESUMEN: El objetivo de este artículo es señalar si el cálculo infinitesimal creado por Leibniz a finales del siglo XVII supone un abandono de la metafísica: mientras que la metafísica se encontraba en la base del fundamento conceptual del cálculo leibniziano, es posible que el éxito de la aplicación del cálculo incitase a los científicos de la época a desgajar la metafísica del corpus científico.
\end{abstract}

PALABRAS CLAVE: Cálculo infinitesimal; Metafísica; Leibniz; Historia de las matemáticas.

ABSTRACT: This paper is intended to examine whether the infinitesimal calculus created by Leibniz at the end of the XVIIth Century implies a withdrawal of metaphysics: although metaphysics provided the conceptual foundation of the leibnizian calculus, it is possible that the success of the calculus incited scientist to separate metaphysics from the core sciences.

KEYWORDS: Infinitesimal Calculus; Metaphysics; Leibniz; History of Mathematics.

\section{El abandono de la metafísica en las ciencias}

El cálculo infinitesimal es uno de los grandes logros científicos

alcanzados por Leibniz a lo largo de su prolífica carrera. Para muchos, especialmente para los estudiosos de las matemáticas, podría decirse incluso que es el mayor logro que alcanzó. Lo cierto es que con la creación del cálculo se marca un antes y un después en el desarrollo de las matemáticas modernas. En este sentido, es sorprendente que el cálculo, por un lado, sea una magnifica herramienta matemática que a su vez, por otro lado, deba sus bases a los planteamientos filosóficos producidos por su creador Leibniz. 
Podría discutirse si la creación de una herramienta tan útil y de belleza tal como el cálculo infinitesimal es realmente fruto de las bases filosóficas de Leibniz, puesto que unos años antes ya Newton había inventado su cálculo de fluxiones. Pero si bien Newton descubrió con anterioridad este cálculo, la calidad conceptual del método newtoniano se encuentra claramente por debajo del leibniziano hasta tal punto que su aplicación no es práctica. Solamente un pensador como Leibniz podría crear una herramienta como el cálculo infinitesimal partiendo de la superación de los infinitos matemáticos, cuestión que posee una clara carga metafísica de la que Leibniz se ocupó con todas sus implicaciones y que estaba conceptualmente fundamentada en base a cuestiones filosóficas.

Y mientras que Leibniz es probablemente el pensador que más ha llevado hasta sus límites el ejercicio de la interdisciplinariedad, de lo cual es muestra este cálculo, en su época el éxito de aplicación que supuso el cálculo facilitaría que los científicos modernos dejasen de dar importancia a las bases filosóficas con las que Leibniz contaba, las cuales fueron responsables de llevarle hasta la creación de este cálculo.

El cálculo presentaba resultados claros para problemas matemáticos y geométricos concretos, y su aplicación no supone apenas esfuerzo mental en comparación con los métodos antiguos. Por ello, para alcanzar estos resultados no es necesario apelar a los fundamentos metafísicos que llevaron a Leibniz a cuestionarse sobre la superación de los infinitos. Es decir: si no es necesario 
apelar a la metafísica para solucionar problemas, ¿para qué acudir a ella? A este paso, que tiene lugar principalmente en el siglo XVII, lo hemos llamado "olvido de la metafísica" y supondría un camino sin retorno en el campo de las matemáticas que a su vez se expandiría hasta cubrir el campo de las ciencias empíricas en general: la metafísica acabaría siendo solamente un problema para filósofos.

Por ello, el objetivo de este artículo es mostrar un ejemplo mediante el cual podemos observar cómo Leibniz aplica con éxito su cálculo infinitesimal, en este caso la discusión sobre la descripción matemática de las curvas mecánicas en la que Leibniz se ve involucrado junto con los hermanos Bernoulli y Huygens en las Acta Eruditorum de 1691, y que ofrece un ejemplo de cómo la aplicación del cálculo, mientras que sitúa a Leibniz en la vanguardia de las matemáticas de su época, marca el camino que tomará la ciencia moderna, que es el de abandonar el estudio de las bases metafísicas que posee.

En este sentido, en la actualidad el ámbito científico no ha cambiado ni en sus ambiciones explicativas ni en su abandono del estudio de la base metafísica, por lo que es menester estudiar el momento de ese abandono y los motivos por los que ocurrió, así como la posibilidad de una vuelta a la interacción entre estas disciplinas.

Por ello nos preguntamos ¿Supone el cálculo infinitesimal un abandono de la metafísica? ¿Hasta qué punto el éxito del cálculo ha facilitado que ese abandono de la metafísica sea un hecho en la actualidad y los signos de 


\section{Dossiê Leibniz, Dissertatio - Volume Suplementar 03 | UFPel [2016]}

recuperación sean mínimos? Para responder a estas cuestiones trataremos el problema de la descripción matemática de las curvas mecánicas y veremos cómo Leibniz aplica el cálculo infinitesimal para superar ese problema. Más adelante hablaremos sobre el abandono de la metafísica en las ciencias y, por último, señalaremos las conclusiones a las que podemos llegar partiendo de lo señalado anteriormente.

\section{El caso de la curva catenaria}

El problema de las curvas mecánicas es un problema heredado de las matemáticas griegas. Ellos ya señalaron que no todas las curvas eran iguales, puesto que unas, fácilmente representables con el uso de la regla y el compás, eran llamadas curvas geométricas; y otras, que estaban generadas por fuerzas físicas, como por ejemplo la gravedad, no podían ser representadas con tal sencillez, a las cuales llamaron curvas mecánicas. Además, mientras que las curvas geométricas podían ser representadas mediante ecuaciones sencillas y con el uso del cálculo geométrico clásico, las mecánicas se resistían a ser representadas mediante un método general.

Descartes, que había llegado a las matemáticas buscando la certeza que la filosofía hasta el momento no le había dado, heredó el problema de las curvas mecánicas. Su ideal era el de la unidad de la ciencia, y por tanto no había motivo por el que excluir unas curvas en geometría, la ciencia que estudiaba la medida 
de los cuerpos, y separarlas de las más complejas. ${ }^{1}$ En este sentido, era capital la búsqueda de un método general que ofreciese la posibilidad de describir este tipo de curvas mecánicas, en lugar de tener que proceder caso por caso para resolverlas. La creación de la geometría analítica de Descartes, mediante la cual se podía resolver analíticamente problemas de geometría y viceversa, ${ }^{2}$ allanó grandemente el camino para que pudiese encontrarse un método general para la descripción matemática de las curvas mecánicas, pero lo cierto es que hasta la aparición del cálculo infinitesimal no hubo ninguna regla que ofreciese un procedimiento general para reducir estas curvas a ecuaciones:

Pues bien, a nadie antes que a Leibniz le vino a la mente la construcción de un algoritmo para este nuevo cálculo, mediante el que se liberara a la imaginación de aquella interminable sujeción a la figuras, que Viète y Descartes habían formulado en su Geometría común o apoloniana, y se alcanzaran cosas más elevadas que pertenecen a la Geometría arquimédea y a aquellas líneas que, llamándolas mecánicas, explícitamente Descartes había excluido del cálculo. Así pues, con el nuevo cálculo de Leibniz, toda la Geometría, en su integridad, queda sometida a un cálculo analítico, y aquellas líneas cartesio-mecánicas, que él ahora llama transcendentes, pueden ser también reducidas a ecuaciones locales mediante la consideración de las diferencias $d x, d d x$, etc y de las sumas, recíprocas a las diferencias, todas ellas introducidas ahora en el cálculo como funciones de

\footnotetext{
1 Descartes (1981), p.29.
}

2 Araña (2015), p.71. 


\section{Dossiê Leibniz, Dissertatio - Volume Suplementar 03 UFPel [2016]}

$x$, cuando antes sólo se utilizaban $x, x x, x^{3}, \sqrt{x, \text { etc }}$ como funciones de cantidades, esto es, potencias y raíces. ${ }^{3}$

El problema de la catenaria surge con Galileo, pasa por el joven Huygens que con sólo 17 años descubre el error del científico italiano y acaba en el cálculo infinitesimal de Leibniz. ${ }^{4}$ Primeramente Galileo había señalado erróneamente que la cadena colgante tomaba la forma de una parábola, pues ambas curvas son muy parecidas. Esta curva a la que Galileo llamó "parábola" es una curva que se forma cuando una cuerda o cadena con masa distribuida de forma uniforme se encuentra sujeta por ambos extremos, y se trata de uno de los tipos de curva mecánica. ${ }^{5}$ Galileo llegó a esta conclusión estudiando el movimiento de los proyectiles y pensó haber demostrado que la curva que presentan toma forma de parábola, es decir, una curva geométrica. Pero se equivocó, puesto que la forma de la cadena colgante está generada por fuerzas mecánicas.

Por otra parte, y según sus propias palabras, Huygens, con tan sólo 17 años ${ }^{6}$, se percató del error de Galileo, tal y como le afirma a Leibniz en una carta el 9 de octubre de 1690:

Mais pour juger mieux de l'Excellence de Vostre Algorithme, j'attens avec impatience de voir les choses que vous aurez trouvées touchant la ligne de

\footnotetext{
3 OFC 7A 405.

4 Trato con mayor profundidad el caso de la catenaria en la correspondencia entre Leibniz y Huygens en Palomo (en prensa).

5 Galileo (1976), '.259.

${ }^{6}$ Aunque en la carta asegura que tenía 15 años, en la fecha de envío de la carta en la que Huygens explica a Mersenne que esta curva no es una parábola, Huygens cuenta con 17 años, ya que nace el 14 de abril de 1629 y la carta tiene fecha de 28 de octubre de 1646. Se trata, sin duda, de un error de Huygens.
} 
la corde ou chaine pendante, que Monsr Bernouilly vous a proposée à trouver; dont je luy scay bon gré, parce que cette ligne renferme des proprietez singulieres et remarquables. Je l'avois considerée autrefois dans ma jeunesse, n'ayant que 15 ans, et j'avois demontré au P. Mersenne, que ce n'estoit pas une Parable, et quelle maniere de pression il faloit pour faire la parabole. $^{7}$

La cadena colgante no podía tomar la forma de una parábola puesto que no podía ser descrita mediante una ecuación sencilla, y así lo mostró Huygens en esta carta escrita el 28 de octubre de 1646 dirigida a Marin Mersenne, amigo de su padre Constantijn. Este hecho mostró sin duda la alta capacidad que poseía el joven Huygens, señalando el error de una de las grandes mentes de la ciencia moderna. Además, Huygens no solamente descubre el error de Galileo sino que además pone nombre a la curva catenaria ya en su madurez en la carta a Leibniz con fecha de 18 de noviembre de 1690, nombre que procede de la palabra latina catena, que significa "cadena". Antes de esta carta, Leibniz, Huygens y todos los intelectuales que estudiaron este problema se referían a la catenaria como la corde pendante o chaine pendante.

A partir de este momento, la cuestión a responder en principio parecía sencilla: si no es una parábola ¿qué forma toma entonces la cadena colgante y cómo describirla matemáticamente? A finales del siglo XVII el problema de la descripción de la catenaria era conocido por todos los matemáticos del momento. Con la idea de probar la eficacia del cálculo creado por Leibniz, Jacob Bernoulli propone en las Acta Eruditorum de 1690 el reto a Leibniz de describir 


\section{Dossiê Leibniz, Dissertatio - Volume Suplementar 03 | UFPel [2016]}

la catenaria en las Acta. El artículo donde se encuentra la proposición de Bernoulli se llama "Analysis problematis antehac propositi, de inventione linea descensus a corpore gravi precurrende uniformiter, fic ut temporibus equalibus equales altitudines emetitur: \& alterius cujusdam Problematis Propositio". ${ }^{8}$ Leibniz acepta el reto de Bernoulli con un artículo titulado "Ad ea, quae vir clarissimus J.B. mense Majo nupero in bis Actis publicavit, Responsio". ${ }^{9}$ Para solucionar el problema de la descripción matemática de la catenaria Leibniz iba a hacer uso de su cálculo infinitesimal y Jacob Bernoulli seguramente estaría al tanto de ello, puesto que él mismo había sido convertido al cálculo leibniziano tras el cálculo de la curva isócrona en mayo de 1690, tal y como el mismo Leibniz señala:

En effet le célèbre Bernoulli, après avoir dans différens problèmes employé avec succès cette Analyse des infinis, s'exprimant par le calcul différentiel, que j'ai contribué à introduire, m'a demandé publiquement dans les Acta de Mai de l'année dernière, p. 218 et suivantes, d'examiner, en en faisant d'épreuve, si notre calcul pouvait s'ètendre à un problème comme celui de la détermination de la Chaînette. ${ }^{10}$

Tan pocas dudas tenía Leibniz sobre la utilidad de este nuevo cálculo que además decidió abrir el reto para que otros matemáticos pudiesen aportar sus propias soluciones al problema. La idea era abrir el plazo de seis meses, luego prorrogado a un año, para que aquellos que quisiesen participar presentasen sus 
soluciones a los editores del Acta Eruditorum para su publicación simultánea. Bajo este panorama, cuatro intelectuales decidieron aportar sus soluciones: Huygens, antiguo maestro de Leibniz en matemáticas, fue uno de ellos. Tambien Tschirnhaus y Johan Bernoulli, con lo que los cuatro mostrarían la eficacia de sus métodos, si bien Tschirnhaus finalmente no llegó a enviar su solución.

Como decía Huygens, esta sería una oportunidad única para mostrar la eficacia del nuevo cálculo de Leibniz. En la correspondencia que mantenían Leibniz y Huygens, durante este periodo discuten acerca de la utilidad del cálculo infinitesimal. El ya antiguo maestro de Leibniz no se encontraba del todo seguro de la utilidad de este nuevo invento leibniziano (al igual que no lo estuvo con el analysis situs, ${ }^{11}$ por ejemplo) y estaba deseoso de poder comparar sus métodos. La impaciencia hizo que Huygens enviase a Leibniz su solución (aunque parcial) antes de su publicación en las Acta Eruditorum mientras le pedía que le enviase también su propia descripción de la catenaria. En la carta del 13 de octubre de 1690, ${ }^{12}$ Leibniz señala que los resultados de ambos solamente se diferenciaban por un signo, aunque no le envió su demostración a Huygens tal y como éste le pedía. En la carta con fecha de 18 de noviembre de $1690,{ }^{13}$ la misma donde Huygens bautiza la catenaria, el holandés señala que seguramente la diferencia de signo se debe al uso de diferentes métodos, y urge a Leibniz a que le envíe su

\footnotetext{
11 Leibniz muestra a Huygens su analysis situs por primera vez el 18 de septiembre de 1679 (AA III 2 840$50)$, y aunque promete grandes aplicaciones y descripciones exactas de cosas naturales como la estructura de las plantas, Huygens no percibe la utilidad de este método y Leibniz lo abandona temporalmente.

12 AA III 4 619-24.

${ }^{13}$ AA III 4 654-8.
} 


\section{Dossiê Leibniz, Dissertatio - Volume Suplementar 03 | UFPel [2016]}

solución completa, algo que Leibniz no llegó a hacer, manteniendo silencio sobre el asunto hasta que en la carta del 2 de marzo de $1691^{14}$ Leibniz le confirmaba a Huygens que John Bernoulli había encontrado una solución y que seguramente el conocimiento de su cálculo le habría ayudado, aunque en su opinión realmente se trataba de un problema que carecía de una complejidad especial. Huygens insistió en dos cartas más para que Leibniz le enviase su solución, pero él no accedió, por lo que tuvo que esperarse a leer directamente las Acta Eruditorum de junio de 1691. Los artículos publicados como parte del reto propuesto por Jacob Bernoulli fueron: Leibniz, "De línea in quam flexile se pondere propio curvat, ejusque usu insigni ad inveniendas quotcunque medias proportionales et logarithmos"; 15 Huygens, "Dynastae in Züchelem, solutio ejusdemproblematis"; ${ }^{16}$ y John Bernoulli, "Solutio Problematis Funicularis, exhibita a Johanne Bernoulli”. ${ }^{17}$ A estos artículos hay que añadir un texto de Jacob Bernoulli que a modo de epílogo cierra las respuestas de Leibniz, Huygens y su hermano Johan. Éste se encuentra dentro del artículo "Specimen alterum calculi differentialis in dimetienda spirali logarithmica, loxodromiis nautarum, et arcis triangulorum sphaericorum; una cum aditamento quodam ad problema funicularum alisquee". ${ }^{18}$ Y por último, Leibniz ofrece además una explicación

\footnotetext{
${ }^{14}$ AA III 5 58-64.

${ }^{15}$ Acta Eruditorum Junio 1691 277-281; GP V 243-247; Leibniz 1989: 186-199.

${ }^{16}$ Acta Eruditorum Junio 1691 281-282; OC 10 95-98; GP V 251-252.

${ }^{17}$ Acta Eruditorum Junio 1691 274-276; GP V 248-250.

${ }^{18}$ Acta Eruditorum Junio 1691 288-290; GP V 252-254.
} 
más detallada de la descripción de la catenaria ofrecida en el reto en las Acta de septiembre del mismo año. ${ }^{19}$

Estos artículos, ya publicados, mostraban a la comunidad científica el avance que suponía el cálculo infinitesimal con respecto a los métodos antiguos, y Leibniz, en correspondencia con Huygens, le habla sobre las diferencias entre los acercamientos de cada uno de ellos. En palabras de Leibniz, tanto él mismo como Bernoulli habían reducido el problema de la descripción matemática de la catenaria al problema de la cuadratura de la hipérbola, puesto que son matemáticamente similares. ${ }^{20} \mathrm{~A}$ pesar de que a posteriori la relación de ambos problemas pueda parecer obvia para el estudioso de hoy en día, Huygens no consiguió, en su aportación, hallar dicha relación, y seguramente, viendo que tanto Leibniz como Bernoulli habían visto claramente que la solución transitaba por el camino del cálculo infinitesimal y él no, siendo el más veterano de los tres, llegó a pensar que había sido víctima de un fraude en el cual Leibniz y Bernoulli habían intercambiado información antes de la publicación de sus artículos en las Acta Eruditorum. ${ }^{21}$ Aunque en la aplicación del cálculo pueden darse diferencias, tal y como Leibniz señala cuando explica las distinciones entre su cálculo de la

\footnotetext{
${ }^{19}$ Acta Eruditorum Septiembre 1691 435-439; GP V 255-258; Leibniz 1989: 200-205.

20 "J'espere que Monsieur Bernoulli fera une plus exacte comparaison; et comme il employe ma methode, je prends part à ce qu'il a fait. Luy et moy nous avons reduit le probleme à la quadrature de l'Hyperbole, nous avons donné tous deux non seulement les tangentes et l'extension de la courbe, mais aussi le centre de gravité de la courbe, et moy j'y ay adjouté le centre de gravité de l'espace. Nous avons donné tous trois les tangentes et l'etendue de la courbe". AA III 5133.

21 “Je veux croire pourtant, puisque vous m'en assurez Monsieur, que vous n'avez point vu la construction de Mr Bernouilly devant que de donner la vostre; mais il se pourroit qu'il seroit venu à vostre connoissance (puisque le memoire de Mr Bernouilli estoit à Leipsich depuis le mois de Decembre, et qu'il n'en avoit pas recommandé le secret) qu'il l'avoit reduite à la quadrature de l'hyperbole" (AA III 5 168).
} 
catenaria y el de Bernoulli, lo cierto es que este nuevo método era lo suficientemente general como para poder describir matemáticamente la catenaria, así como otras curvas mecánicas, con mucho menor esfuerzo que con el uso de los métodos antiguos.

\section{El éxito del cálculo y sus consecuencias para la metafísica}

Como hemos comprobado, en principio el cálculo de Leibniz ciertamente no culmina en cuestiones filosóficas, sino que se trata de una herramienta para obtener resultados. En ese sentido, el cálculo supone un abandono de las cuestiones metafísicas en el sentido de que, aunque parte de ellas, éstas no son necesarias para su aplicación en casos como puede ser el del problema de la catenaria. Además, los resultados alcanzados mediante la aplicación del cálculo son proposiciones verdaderas, puesto que se han alcanzado mediante principios lógicos. Todo ello no quiere decir que el nuevo cálculo leibniziano carezca de algún carácter filosófico, puesto que, como dice Arana, la "epistemología logicista [de Leibniz] se articula y define según la inspiración de descubrimientos conceptuales procedentes del cálculo infinitesimal". 22

Por lo cual no podemos precipitarnos al pensar que la metafísica que rodea al cálculo infinitesimal se encuentre solamente en las bases de su creación.

${ }^{22}$ Arana (2015), p.123. 
De hecho, Leibniz comienza el artículo publicado en las Actas Eruditorum de junio de 1691 diciendo que hay dos aplicaciones para el problema de la catenaria: una, comprender la utilidad del análisis, y otra, hacer progresar el arte de la construcción. $^{23}$ La utilidad del análisis queda patente con el caso de la descripción de la catenaria, pero, ¿qué hay del arte de la construcción? Para comprender este punto debemos atender a la praxis científica de Leibniz, la cual contrasta grandemente con la de otros intelectuales de la época moderna, como Huygens o Descartes. Sobre Huygens afirma Leibniz que no cultivó el gusto por la metafísica y en el mismo lugar Leibniz habla también de Descartes, de quien dice que su filosofía es la antesala de la verdad, la cual se alcanzará eventualmente. ${ }^{24}$ ¿Qué significa esto? Que aunque el cálculo no acaba en consecuencias filosóficas, para Leibniz, puesto que todas las disciplinas estaban interrelacionadas, sí que implicaba consecuencias a posteriori. Haciendo matemáticas, Leibniz busca la efectividad, alejándose de discusiones filosóficas que impidan alcanzar las verdades sencillas. Pero eso no significa que las matemáticas se encuentren alejadas de la metafísica, y es que son estas especulaciones filosóficas, como el intento de la superación de los infinitos, los que permiten la aparición del cálculo leibniziano. Y en cuanto a las consecuencias en sí, el cálculo infinitesimal forma parte de un corpus de disciplinas en el que todas conjuran para alcanzar las verdades últimas. Las

${ }^{23}$ Acta Eruditorum Junio 1691 277; GP V 243; Leibniz (1989), p.186.

24 GP III 607. 


\section{Dossiê Leibniz, Dissertatio - Volume Suplementar 03 | UFPel [2016]}

matemáticas ya no son un modelo a imitar, como Descartes pensaba, sino que son una herramienta efectiva para alcanzar verdades que forman parte central del corpus de la ciencia universal, ${ }^{25}$ y que media con la teología, la metafísica, la filosofía moral y la física. Para Leibniz de hecho, el cálculo es útil y esencial para el avance de las matemáticas y, por lo tanto, también del resto de las ciencias:

Premierement : si les definitions des figures se peuvent exprimer
parfaitement par ce calcul, toutes les proprietés se peuvent trouver par ce
calcul ; secondement puisque la definition de la spherique, du plan du cercle
et de la droite se peuvent parfaitement exprimer par le calcul; les definitions
des autres lieux ou figures se pourront exprimer aussi, car leurs points se
trouvent tousjours par des droites ou circulaires. Troisement si tous les lieux
et leur proprietés se peuvent exprimer et trouver par de calcul ; toutes les
figures planes ou solides, et toutes les situations, et leur changemens c'est-
à-dire les mouvemens se pourront exprimer aussi, et par consequent on
pourra expliquer les machines mêmes sans tracer des figures, et on les
pourra inventer sans charger l'imagination. ${ }^{26}$

En el proyecto de la ciencia universal Leibniz aunaría todas las disciplinas existentes, y al hacerlas cooperar de un modo transversal podrían dar respuesta a todas las cuestiones existentes posibles. Esta praxis leibniziana resalta sobre la del resto de los intelectuales de la época, que acostumbraban a situar unas disciplinas por encima de otras. En esta búsqueda de la unión e interacción de disciplinas radica el optimismo leibniziano, mediante el cual se puede llegar a afirmar la verdad de las cosas, que en última instancia depende de Dios. 
Si bien la idea de Leibniz era formalizar esta ciencia universal, sus avances en matemáticas comienzan a tener una importancia capital a ojos del resto de los intelectuales de la edad moderna con el éxito de aplicación del cálculo infinitesimal. De hecho, es la solución de Leibniz al problema de la catenaria la que justifica a la comunidad académica, y especialmente a Huygens, el uso y la utilidad de su cálculo infinitesimal, con el cual se presentaba un método general con el que describir matemáticamente las curvas mecánicas. Sin embargo, a pesar de la importancia en Leibniz de la interacción de las matemáticas, la física y la metafísica para desvelar la verdad, a lo largo de los artículos que forman parte de la discusión sobre la catenaria no se denota el uso de ningún razonamiento metafísico, ya que se trata de una demostración matemática. Y puesto que no es necesaria la metafísica para el uso y aplicación del cálculo, seguramente la necesidad de la base metafísica con la que Leibniz contaba, y que era esencial para el proyecto de la ciencia universal, no fue correctamente transmitida al resto de los matemáticos de la época. A ello hay que añadir la creación análoga del cálculo por parte de Newton, quien en su sistema general prescinde no solamente de una explicación metafísica de los fenómenos físicos sino incluso de la explicación mecánica de estos fenómenos, como por ejemplo de la gravedad, de la cual solamente necesitaba saber que es una cualidad que tienen los cuerpos.

Por ello, en el proceso de formalización de la ciencia universal se plantea una cuestión capital: ¿es necesaria la metafísica para llegar al cálculo? En 


\section{Dossiê Leibniz, Dissertatio - Volume Suplementar 03 | UFPel [2016]}

Leibniz fue necesaria para llegar a su creación incluyendo su excelente forma de notación matemática, y no solamente eso, sino que sin la metafísica el corpus de la ciencia universal carecería de sentido, puesto que éste requiere de la participación de todas las ciencias con las matemáticas como punto central. Pero, sin embargo, el cálculo supone un abandono de la metafísica en el sentido de que se puede aplicar independientemente, a ojos de la comunidad científica, sin la necesidad de apelar a ningún tipo de principio filosófico. Por ello, mientras que Leibniz llega hasta la creación del cálculo infinitesimal gracias a sus inquietudes filosóficas, así como gracias a la superación de cuestiones matemáticas de calado metafísico como es la superación del infinito matemático, la comunidad científica, que se centra más en la consecución de resultados que en las implicaciones trascendentales de estos resultados, desgaja el cálculo infinitesimal del proyecto de la ciencia universal, y se produce un abandono de la metafísica que marca el camino que recorrerán las disciplinas científicas hasta nuestros días.

\section{Conclusiones}

Mientras que en Leibniz el éxito de la aplicación del cálculo infinitesimal, del que hemos podido estudiar el caso de las curvas mecánicas, no supone un abandono de la metafísica sino que supone más bien un empuje e inspiración para sus principios metafísicos y su epistemología, sí que facilita que los matemáticos de la edad moderna hagan una separación de la metafísica con 
respecto a la aplicación del cálculo y por tanto impidan que el testigo de la interacción transversal de disciplinas al modo leibniziano pase a futuras generaciones. Por ello, llevan a cabo un abandono de la metafísica que culminará con el éxito de propuestas como la de Newton con sus Principia Mathematica y cuyas consecuencias todavía hoy están presentes en las disciplinas científicas.

Por ello, mientras que Leibniz promueve un ejercicio de la interdisciplinariedad para llevar adelante la empresa de la razón, dicho ejercicio quedará anulado durante decenas de años hasta la reflexión sobre la necesidad de la interacción de disciplinas aparezca en nuestros días. Sin embargo, el tipo de interdisciplinariedad que pueda existir hoy día en las ciencias supone un ejercicio de interdisciplinariedad sesgada, puesto que en ellas todo debe reducirse a la física, a la química, a la biología o a la psicología y la utilización de otras disciplinas sirve solamente como apoyo y no como parte fundamental de la explicación de los fenómenos.

En nuestros días es necesario reabrir el debate de la unidad de la ciencia, puesto que la aplicación de disciplinas sin la interacción transversal que buscaba Leibniz ha llevado a las ciencias a toparse con el límite de sus pretensiones cognoscitivas, y a todas luces son incapaces de dar cuenta de fenómenos como la aparición de la consciencia o el origen de la vida. Ello contrasta grandemente con el optimismo metafísico de Leibniz, mediante el cual 
se podría "reconstruir el camino seguido por aquella [la Voluntad creadora de Dios] hacia la perfección, hacia el mejor de los mundos posibles". ${ }^{27}$

Por tanto, ante todo ello surgen varias preguntas que sería pertinente responder en futuras ocasiones: cha sido el abandono de la metafísica en las ciencias una necesidad para el éxito de estas disciplinas? ¿Es posible enmendar ese camino que han tomado las ciencias empíricas desde aquel siglo? ¿Es, acaso, algo deseable?

\section{Ediciones de escritos de Leibniz}

LEIBNIZ, G.W. (1923-), Sämtliche Schriften und Briefe, herausgegeben von der Deutschen Akademie der Wissenschaften, Darmstadt, 1923; Leipzig, 1938; Berlin, 1950 y prosigue (Citado como AA). (1875), Die philosophischen Schriften, herausgegeben von C. I. Gerhardt, 7 Bände, Berlin 1875-1890 (Citado como GP). (1849), Die mathematischen Schriften, herausgegeben von C. I.

Gerhardt, 7 Bände, Berlin (1849-1863). (Citado como MS).

(2007-), Obras filosóficas y científicas, editada por la Sociedad

Española Leibniz, director de la edición: Juan Antonio Nicolás, Granada, Editorial Comares (citada como OFC y volumen). 

(1989), La naissance du calcul différentiel : 26 articles des "Acta

Eruditorum". Vrin : Paris.

\section{REFERENCIAS BIBLIOGRÁFICAS}

ARANA, J. (2015), El proceso bistórico de separación entre ciencia y filosofia, Madrid: Real Academia de Ciencias Morales y Políticas.

BOYER, C. B. (1949), The History of the Calculus and Its Conceptual Development, New York: Dover Publications.

DeSCARTES, R. (1981), Discurso del Método. La Dióptrica. Los Meteoros. La Geometría, Madrid: Alfaguara.

GALILEO, G. (1976), Consideraciones y demostraciones matemáticas sobre dos nuevas ciencias, Madrid: Editora Nacional.

HUYGENS, C. (1888-1950), Oeuvres complètes de Christiaan Huygens, 22 vols., Martinus Nijhoff, The Hague.[Citado como OC]

PALOMO, M. (en prensa), “The Mathematical Description of the Catenary Curve on the Correspondence Between Leibniz and Huygens”, Fichant, M., Pisano, R., Oliveira, A. R. E. y Bussotti, P. (Eds.), Homage to Gottfried Wilhelm Leibniz as Scientist and Engineer 1646-2016. New Scientific and Epistemological Insights, Dordrecht: Springer. 